\title{
A review of haemostasis following minor oral surgery procedures
}

\begin{abstract}
This paper focuses on the process of haemostasis and methods of aiding haemostasis following minor oral surgery; these include both local and systemic measures. Consideration is given to patients with bleeding disorders, on anticoagulant medication or antiplatelet medication. The importance of recognising the increased risk of haemorrhage following minor oral surgery in such patients is explored with current recommendations for good practice in such patients. The dilemma as to whether antiplatelet and anticoagulant medication should be continued or discontinued prior to or immediately following minor oral surgery procedures is also discussed.
\end{abstract}

Volume 7 Issue I - 2017

\author{
Basmal Ria,' Emma Wates,' Sama Ria ${ }^{2}$ \\ 'Department of Oral and Maxillofacial Surgery, St George's \\ Hospital, UK \\ ${ }^{2}$ Oral and Maxillofacial Surgery, Northwick Park Hospital, UK
}

Correspondence: Basmal Ria, Department of Oral and Maxillofacial Surgery, St George's Hospital, NHS Trust, UK, Email basmalria@live.com

Received: April 06, 2016 | Published: April 19, 2017

\section{Clinical relevance}

Haemorrhage following minor oral surgery is to be expected; if left uninterrupted and persistent, can prove fatal. It is therefore essential to understand the process of haemostasis and methods one can use to aid haemostasis in such instances. Similarly, with a shift in population demographics and an increase in life expectancies, comes a surge in co-morbidities and new medication to tackle them; among them anticoagulant and antiplatelet medication. It is important to be aware of how these medications can affect our patients, and how to manage such patients to avoid unnecessary prolonged haemorrhage following minor oral surgery.

\section{Objective}

To explain the process of haemostasis and the different methods of aiding haemostasis following minor oral surgery procedures. To understand the importance of aiding haemostasis in patients with a bleeding disorder or on anticoagulant or anti-platelet medication and the role of secondary care.

\section{Introduction}

\section{Understanding postoperative haemorrhage}

Persistent postoperative haemorrhage may result from poor or inadequate haemostasis. Lockhart et al. ${ }^{1}$ defines clinically significant post-extraction haemorrhage to include persistent bleeding for over 12 hours or one which prompts a patient to return to the dental practice or accident and emergency or those which cause large haematomas or may require a blood transfusion. ${ }^{1}$ Post-extraction haemorrhage is commonly classified as Primary, Reactionary and Secondary haemorrhage; the former occurs at the time of surgery, while reactionary haemorrhage occurs 2-3 hours post-operatively due to cessation of vasoconstriction and the latter occurs up to 2 weeks post-operatively commonly due to infection. ${ }^{2}$ The aim of this article is to review the physiological process of haemostasis and to explore how common disorders and drugs may affect this process; and how we should be managing these patients.

\section{Understanding the haemostasis pathway}

Haemostasis is achieved through a balance of haemostasis (clot formation) and fibrinolysis (clot breakdown) resulting in a clot, the former involves 3 stages: vascular spasm, platelet plug formation and coagulation. Vascular spasm involves prompt constriction of a damaged blood vessels to prevent blood loss. This is encouraged by pain receptors, smooth muscle injury as well as platelets which release serotonin, a vasoconstrictor. This is followed by formation of the platelet plug triggered by exposed collagen in the walls of damaged blood vessels. This is also known as primary haemostasis where platelets adhere to damaged vessel walls as well as one another in an attempt to form a plug. Platelets are produced in the bone marrow and degranulate to release substances to encourage the next stage of the coagulation cascade. The coagulation (clotting) cascade follows to finalise the process of haemostasis and includes the activation of a series of clotting factors, usually produced by the liver, to bring about the formation of a clot; this is achieved via an intrinsic and extrinsic pathway and is often referred to as secondary haemostasis. The extrinsic pathway is quicker with fewer steps, taking only seconds; it is triggered by substances released by damaged tissue which result in the activation of factors VII, V and X. The intrinsic pathway however involves the activation of factors XI to IX to VIII to X and is triggered by platelets and therefore can take longer up to a few minutes. Both pathways result in the activation of factor $\mathrm{X}$ and subsequently the formation of thrombin and subsequently fibrin and thus a clot. ${ }^{3}$

This is the first stage of wound healing and crucial knowledge for any practitioner undertaking an invasive procedure.

\section{Local measures to aid haemostasis following minor oral surgery}

It is important to make note of the site and origin of the haemorrhage, be it soft tissue, bone or originating from the vasculature as will impact management. If soft tissue in origin, investigate for local infection or tears. If the former, the area must be cleaned, any infected or necrotic tissue removed. If the latter, a tear, an attempt should be made to salvage with suturing. If the origin of the bleed is 
bone, other methods including bone wax may need to be considered; these are discussed below.

\section{Digital pressure}

Routinely, following the removal of teeth local measures are undertaken to assist haemostasis; this includes application of digital pressure to cover the socket for a few minutes, commonly with saline dampened sterile gauze to prevent adherence to the wound/ socket. This is traditionally sufficient for initial haemostasis and clot formation (Figure 1). Clear post-operative instructions should be also be given as failure to care for the area appropriately may result in infection or secondary haemorrhage. ${ }^{4}$

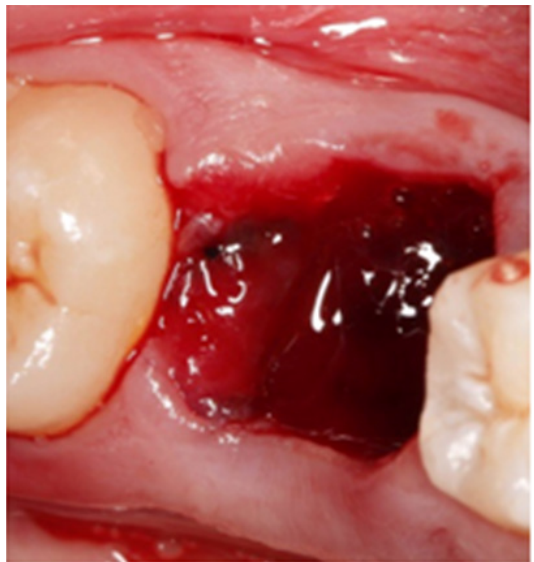

Figure I UR6 clot formation following digital pressure.

\section{Haemostatic agents: tranexamic acid, ferric sulphate and silver nitrate}

For most patients local measures suffice, if not haemostatic agents may be considered. These include tranexamic acid, ferric sulphate and silver nitrate. Tranexamic acid may come in the form of a mouthwash and can be effective in controlling postoperative bleeding. ${ }^{5}$ It acts by binding to plasminogen and inhibiting subsequent fibrinolysis. The $\mathrm{BCSH}$ recommend 5\% tranexamic acid mouthwashes used four times a day for two days following dental surgery on patients taking oral anticoagulants such as warfarin. ${ }^{6}$ Ferric Sulphate is a well-established dental haemostatic agent commonly used as an astringent to arrest gingival bleeding but some studies have found it to be associated with adverse reactions inflammation and delayed osseous healing and therefore practitioners would be wise to use with care. ${ }^{7}$ Silver nitrate can also be used to aid haemostasis following minor oral surgery.

\section{Adrenaline}

Tranexamic acid is not readily available in most primary care settings and as such, adrenaline may prove to be a helpful alternative. It is readily available in most dental practices and quite often in dental local anaesthetics. It acts as a vasoconstrictor, limiting blood flow to the area and contributing to the first stage of haemostasis. Similar to tranexamic acid, it may be used to dampen gauze and held at the extraction site postoperatively to arrest bleeding.

\section{Surgicel and suture}

Surgicel is a topical resorbable haemostatic agent consisting of oxidised cellulose which breaks down in moisture to lower the $\mathrm{pH}$ and cause localized vasoconstriction. Its main haemostatic effect comes from its matrix which encourages platelet adhesion, accelerating platelet plug formation and subsequent fibrin clot formation. ${ }^{8}$ It is often placed into dental extraction sockets postoperatively to aid haemostasis with or without sutures. In the authors' opinions a 3.0 resorbable braided type suture with a $1 / 2$ circle such as vicryl following a horizontal or cross-mattress suture technique is recommended to help retain the surgicel within the socket for a week until its resorption, as well as contract the expanded socket to aid haemostasis (Figure $2 \&$ $3)$. Sutures are also recommended for soft tissue tears and following more invasive surgical extractions involving mucoperiosteal flaps to help replace and secure flaps postoperatively and aid haemostasis and wound healing.

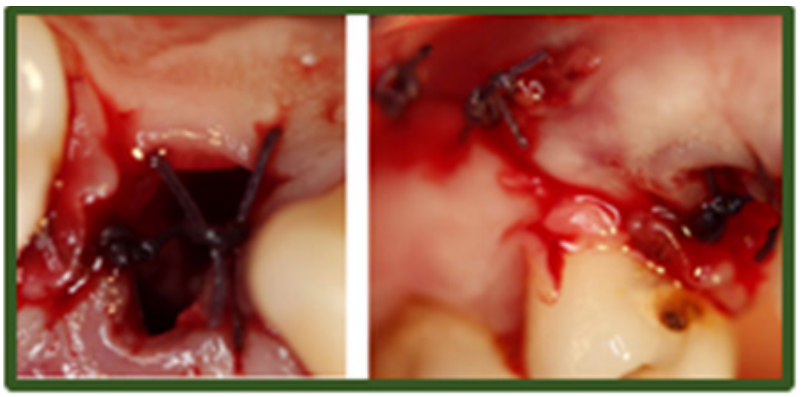

Figure 2 Mucoperiosteal flap replaced following surgical removal of UR4 Distal relieving incision closed with simple interrupted sutures and surgical and cross-mattress suturing of UR4 socket.

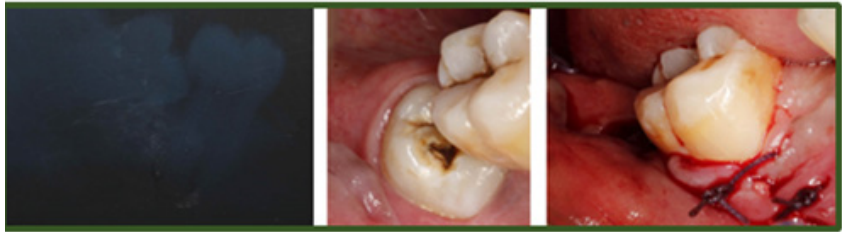

Figure 3 A: Long cone Periapical of mesioangular impacted LR8; B Mesioangular LR8 in situ pre-operatively; C: Mucoperiosteal flap replaced following surgical removal of LR8. Mesial relieving incision closed with simple interrupted sutures and surgical and cross-mattress suturing of LR8 socket.

\section{Gelatin sponges}

Haemostatic resorptive gelatin sponges such as Gelotamp can be effective in controlling post-extraction bleeding as well as minimizing other complications such as infection and dry socket. ${ }^{9}$ These are used similarly to surgicel and placed into the socket postoperatively with or without suture placement.

\section{Bone wax}

Bone wax is a non-absorbable mixture of beeswax, paraffin, isopropyl palmitate, and a wax-softening agent which aids haemostasis by occluding bleeding channels in bone with a tamponade effect. Although easy to handle, relatively inexpensive and fast to act, it is not absorbed by the body and can impair bone healing ${ }^{10}$ and as such should be used with caution. If used, it may be removed following haemostasis and avoided in areas of contamination as if left in situ can result in bone granuloma formation and may serve as a hub for infection. ${ }^{11}$

\section{Vascular haemorrhage: cautery, ligation and electrocautery}

Vascular haemorrhage may cause extensive bleeding and subsequent distress to patients who should be reassured. Although 
not common practice in primary care, cautery and ligation play a role in haemostasis postoperatively. Ligation is often used to arrest haemorrhage originating from larger blood vessels and cautery often used for smaller blood vessels. ${ }^{4}$ Electrocautery involves sealing a damaged vessel with heat while ligation involves tying off a vessel with suture. ${ }^{4}$ If the practitioner does not have either the appropriate equipment or experience, it is important to refer these patients to the maxillofacial or emergency department for immediate care. Ligation of main feeder vessels such as the facial, lingual internal and external carotid arteries can prove lifesaving if both local and systemic measures fail to arrest haemorrhage following minor oral surgery. ${ }^{12}$

\section{Systemic measures to aid haemostasis following minor oral surgery}

Where haemorrhage persists and local measures have failed, systemic measures may be employed to achieve haemostasis; these are frequently employed in secondary care settings. Systemic measures include supplemental tissue factors and fresh frozen plasma. If bleeding is still not arrested or the patient suffers extensive blood loss, a blood transfusion may then be considered to avoid fatality and angiography/ neuroradiology may be considered. ${ }^{12}$ Blood tests including a full blood count, coagulation screen and group and save are taken to help screen for undiagnosed coagulation disorders and other potential causes. Post-extraction haemostasis can be affected by a number of medical conditions; these include both hereditary and acquired disorders such as haemophilia, von willebrands disease, thrombocytopenia, leukaemia, hypertension, liver dysfunction, alcoholism and conditions requiring anticoagulant or antiplatelet medication. This emphasises the importance of taking a thorough medical history prior to considering minor oral surgery.

\section{Bleeding disorders: haemophilia and Von Willebrand disease}

Haemophilia is an inherited bleeding disorder categorised into type $\mathrm{A}$ and B; the former associated with deficiency in factor VIII, the latter associated with deficiency in factor IX. Contrary to popular belief, these patients only bleed for a longer time but do not bleed more profusely when compared to non-haemophiliacs. ${ }^{13}$ Von Willebrand (VW) disease is the commonest congenital bleeding disorder and is associated with a deficiency/abnormality in von Willebrand factor which plays a role in both stabilising factor VIII and allowing platelets to adhere to blood vessel walls. This is critical in haemostasis and subsequently these patients are also at risk of extensive postoperative bleeding following tooth extraction. ${ }^{13}$

\section{Achieving haemostasis in patients with bleeding disorders}

Patients with congenital bleeding disorders are more likely to experience significant bleeding following minor oral surgery procedures. Overall postoperative haemorrhagic management of these patients can vary depending on the condition, severity and treatment procedure. It is crucial to liaise with haematologists prior to considering invasive procedures. This can help minimise the need for coagulation factor replacement therapy ${ }^{13}$ and desmopressin therapy which encourage the release of factor stores in haemophilia A and VW disease. The latter incudes anti-fibrinolytic tranexamic acid which may be administered either locally as a mouthwash, systemically, or in combination and can be effective in aiding haemostasis in haemophiliacs. ${ }^{13,14}$ Administering factor replacement therapy should be given as near as possible to the time of minor oral surgery and can be more effective if in combination with anti-fibrinolytic therapy than either alone. ${ }^{14}$ Local haemostatic measures also help achieve haemostasis in these patients ${ }^{14}$ and there is evidence to support the use of oral tranexamic acid in combination with tranexamic mouthwash to aid haemostasis. ${ }^{13,14}$

\section{Anticoagulants: warfarin}

Warfarin is an anticoagulant commonly prescribed for the treatment and prevention of arterial and venous thrombosis. ${ }^{15}$ It acts by inhibiting the interconversion of vitamin $\mathrm{k}$ and thus the production of clotting factors in the liver. ${ }^{16}$ Patients on warfarin are at an increased risk of post-extraction bleeding. ${ }^{17}$ However, temporarily discontinuing anticoagulants can put patients at risk of thrombosis with potentially fatal consequences. ${ }^{18}$ Local measures are effective in controlling most postoperative bleeding following minor oral surgery in patients with an INR upto 4.0. ${ }^{17}$ The risk of significant postoperative bleeding in patients on oral anticoagulants with a stable INR in the therapeutic range 2-4 is very small and as such, the overall recommendation by the $\mathrm{BCSH}$ is not to discontinue oral anticoagulants prior to tooth extraction for most patients. It would be prudent to consult the General Practitioner or Haematologist prior to tooth removal if the INR exceeds 4.0. ${ }^{19}$

Achieving haemostasis in the warfarinised patient: Management of such patients should include a pre-operative INR check, at least 72 hours prior to minor oral surgery. ${ }^{19}$ Haemostasis is often achieved with local measures, including intra-operative placement of surgicel/ gelatin sponges with suturing and postoperative tranexamic acid mouth wash prescriptions and avoiding NSAID analgesics due to their anti-platelet action and risk of further haemorrhage. ${ }^{19}$ patients on oral anticoagulants with comorbidities such as liver or renal disease, thrombocytopenia or also taking anti-platelet drugs are at an even greater risk of postoperative bleeding and should have oral surgery procedures undertaken in a secondary care setting.

Antiplatelets: Antiplatelets act by inhibiting platelet aggregation. Electing to discontinue a patient's antiplatelet medication can have detrimental effects including thromboembolic events an average of 10 days following cessation. ${ }^{20}$ Literature show antiplatelet monotherapy has no significant effect on post-extraction bleeding and any bleeding should be sufficiently managed with local measures. ${ }^{21}$ It is not uncommon for patients to have a combination therapy of antiplatelet medication, including combinations or aspirin, clopidogrel or dipyridamole. These have a synergistic effect when combined ${ }^{22}$ and increase the risk of postoperative bleeding and may require referral to secondary care services for oral surgery procedures. Similarly nonsteroidal anti-inflammatory analgesics (NSAIDs) effect platelet aggregation and should be avoided in such patients to reduce the risk of postoperative haemorrhage. ${ }^{23}$

Newer oral anticoagulants (NOAC's): The new oral anti-coagulants are increasingly prescribed by our medical colleagues; these include dabigatran, a direct thrombin inhibitor, and rivaroxaban, a factor Xa inhibitor. Their rapid onset short half-life means they do not require monitoring and at present lack a reversal agent. ${ }^{24}$ Current consensus is to allow patients to continue their NOAC therapy when undergoing routine minor oral surgery procedures without altering the dose in patients with normal renal function. ${ }^{24}$ Oral and maxillofacial expert opinion is varied and currently there is conflicting advice on managing patients on NOAC therapy, therefore it is good practice to consult 
a haematologist and local hospital guidelines should be followed However, temporary cessation of dabigatran should be considered at least 24 hours prior to extensive oral or maxillofacial surgery, after liaising with the patient's GP and haematologist, and can be recommenced postoperatively once a stable clot has formed. ${ }^{24}$ If such patients are at an increased risk of thromboembolism, perioperative bridging anticoagulation with subcutaneous low molecular weight heparin is recommended. ${ }^{24}$

\section{Conclusion}

With a shift in population demographics and an increasingly aging population comes an increase in comorbidities and polytherapy including anticoagulant therapy. As such, it is important practitioners are aware of how to manage such patients when undertaking minor oral surgery procedures and should be aware of a patients expected therapeutic INR range as this can vary. While most patients have a therapeutic INR range of 2.0-3.0 (below 4.0) and haemostasis routinely achieved with local aids, with advances in health care and an increase in life expectancies, increasingly patients with prosthetic heart valves present for minor oral surgery procedures. These patients have a higher therapeutic INR range which can exceed 4.0; as such it would be wise to undertake oral surgery procedures in a secondary care setting with advice from other healthcare professionals including a haematologist, cardiologist, oral surgeon and general practitioner. With the introduction of NOAC's and the difficulty in monitoring their effects, meticulous communication with other healthcare professionals including general practitioners and haematologists is paramount. Although literature surrounding rarer congenital bleeding disorders and newer oral anticoagulants remains limited at large, guidelines are regularly revised to enhance patient care and it is the practitioner's responsibility to ensure they are up to date with these changes prior to undertaking minor oral surgery procedures in these patients.

\section{Acknowledgments}

None.

\section{Conflicts of interest}

The authors declare there is no conflict of interest.

\section{References}

1. Lockhart PB, Gibson J, Pond SH, et al. Dental management considerations for the patient with an acquired coagulopathy. Part 1: Coagulopathies from systemic disease. Br Dent J. 2003;195(8):439-445.

2. McCormick NJ, Moore UJ, Meechan JG. Haemostasis part 1: The management of post-extraction haemorrhage. Dent Update. 2014;41(4):290-296.

3. Kenneth Saladin. Anatomy \& Physiology. The Unity of Form and Function. 5th ed. New York, USA: McGraw-Hill Education; 2014:11248.

4. McCormick NJ, Moore UJ, Meechan JG. Haemostasis part 1: The management of post-extraction haemorrhage. Dent Update. 2014;41(4):290-296.

5. Blinder D, Manor Y, Martinowitz U, et al. Dental extractions in patients maintained on continued oral anticoagulant: comparison of local haemostatic modalities. Oral Surg Oral Med Oral Pathol Oral Radiol Endod. 1999;88(2):137-140.
6. Perry DJ, Noakes TJC, Helliwell PS. Guidelines for the management of patients on oral anticoagulants requiring dental surgery. $B D J$. 2007;203:389-393.

7. Lemon RR, Steele PJ, Jeansonne BG. Ferric sulfate hemostasis: effect on osseous wound healing. Left in situ for maximum exposure. $J$ Endod. 1993;19(4):170-173.

8. Achneck HE, Sileshi B, Jamiolkowski RM, et al. A comprehensive review of topical hemostatic agents: efficacy and recommendations for use. Ann Surg. 2010;251(2):217-228.

9. Cai YH, Lu CS. A clinical study of gelatamp colloidal silver gelatin sponge on preventing the complication of teeth extraction. Hua Xi Kou Qiang Yi Xue Za Zhi. 2008;26(5):519-521.

10. Brightmore TG, Hayes P, Humble J, et al. Haemostasis and healing following median sternotomy. Langenbecks Arch Chir. 1975:39-41.

11. Johnson P, Fromm D. Effects of bone wax on bacterial clearance. Surgery. 1981;89(2):206-209.

12. Hassan G Moghadam, Marco F Caminiti. Life-threatening hemorrhage after extraction of third molars: case report and management protocol. $J$ Can Dent Assoc. 2002;68(11):670-674.

13. Anderson JAM, Brewer A, Creagh D, et al. Guidance on the dental management of patients with haemophilia and congenital bleeding disorders. BDJ. 2013;215:497-504.

14. Hermans C, Altisent C, Batorova A, et al. Replacement therapy for invasive procedures in patients with haemophilia: literature review, European survey and recommendations. Haemophilia. 2009;15(3):639658.

15. Hirsh J. Oral anticoagulant drugs. N Engl J Med. 1991;324:1865-1880.

16. Hirsh J, Dalen J, Anderson DR, et al. Oral anticoagulants: mechanism of action, clinical effectiveness and optimal therapeutic range. Chest. 2001;119(1):8S-21S.

17. Hiroshi Iwabuchi, Yutaka Imai, Soichiro Asanami, et al. Evaluation of postextraction bleeding incidence to compare patients receiving and not receiving warfarin therapy: a cross-sectional, multicentre, observational study. BMJ Open. 2014;4(12):1-9.

18. Wahl MJ. Dental surgery in anticoagulated patients. Arch Intern Med. 1998;158(15):1610-1616.

19. Perry DJ, Noakes, Helliwell PS. Guidelines for the management of patients on oral anticoagulants requiring dental surgery. $B D J$. 2007:389393.

20. Biondi-Zoccai GL, Lotrionte M, Agostoni P, et al. A systematic review and meta-analysis on the hazards of discontinuing or not adhering to aspirin among 50279 patients at risk for coronary artery disease. Eur Heart J. 2006;27(22):2667-2674.

21. Krishnan B, Shenoy N, Alexander M. Exodontia and antiplatelet therapy. Journal of Oral and Maxillofacial Surgery. 2008;66(10):2063-2066.

22. Harder S, Klinkhardt U, Alvarez JM. Avoidance of bleeding during surgery in patients receiving anticoagulant and/or antiplatelet therapy. Pharmacokinetic and pharmacodynamic considerations. Clin Pharmacokinet. 2004;43(14):963-981.

23. Surgical management of the primary dental care patient on antiplatelet medication, UKMI NHS North West Medicines information centre. 2007.

24. O'Connell JE, Stassen L. New oral anticoagulants and their implications for dental patients. Journal of the Irish Dental Association. 2014;60(3):137-143. 